FORMATION Formation emploi

Revue française de sciences sociales

106 | avril-juin 2009

Pêle-mêle

\title{
Faut-il encore et toujours désespérer de la relation formation-emploi?
}

Dialogue avec Paul Santelmann

Philippe Trouvé

\section{(2) OpenEdition \\ Journals}

Édition électronique

URL : http://journals.openedition.org/formationemploi/1958

DOI : 10.4000/formationemploi.1958

ISSN : 2107-0946

\section{Éditeur}

La Documentation française

Édition imprimée

Date de publication : 1 juin 2009

Pagination : 75-87

ISSN : 0759-6340

\section{Référence électronique}

Philippe Trouvé, « Faut-il encore et toujours désespérer de la relation formation-emploi ? », Formation emploi [En ligne], 106 | avril-juin 2009, mis en ligne le 01 juin 2011, consulté le 30 octobre 2020. URL : http://journals.openedition.org/formationemploi/1958; DOI : https://doi.org/10.4000/ formationemploi.1958 


\title{
Faut-il encore et toujours désespérer de la relation formation-emploi ? Dialogue avec Paul Santelmann
}

\author{
Par Philippe Trouvé
}

\section{P. Trouvé commente l'article de P. Santelmann "Les distorsions diplômes/ qualifications: I'exemple des techniciens et agents de maîtrise de type industriel », paru dans le numéro 105 de Formation Emploi.}

Le moins que l'on puisse dire est que le thème des dysfonctionnements de la relation formationemploi (ou «diplômes/qualifications »), abordé par Paul Santelmann, ne fait pas partie de ceux qui, dans la longue tradition du Céreq, ont été les plus négligés. En attestent trois livraisons copieuses parmi les plus récentes qui ont tenté, chacune à leur manière, d'illustrer les accumulations de connaissances dans le domaine, et qu'il serait bon de ne pas trop rapidement évacuer avant d'en avoir tiré toutes les leçons, soit: Giret, Lopez, Rose (2005), le numéro anniversaire 101 de Formation Emploi (2008) et Paul, Rose (2008).

Il fallait donc s'y attendre, la thèse principale soutenue par P. Santelmann n'est pas totalement originale: depuis trois décennies, l'écart entre l'évolution des qualifications professionnelles dans l'appareil de production et celle des diplômes produits par le système éducatif n'a pas cessé de se creuser. À la «complexification technico-organisationnelle des contenus $d u$ travail » aurait répondu massivement un mot d'ordre simpliste et quasi consensuel d'élévation générale de la formation initiale, accen- tuant non seulement la sélectivité à l'entrée des jeunes dans l'emploi et la relégation des enseignements professionnels de niveaux $\mathrm{V}$ et $\mathrm{IV}^{1}$, mais aussi

\footnotetext{
${ }^{1}$ Selon la Nomenclature des niveaux de formation adoptée dès la fin des années 60, le niveau VI correspond à l'absence de diplôme, le niveau $\mathrm{V}$ aux Certificat d'aptitude professionnelle (CAP) et Brevet d'études professionnelles (BEP), le niveau IV aux Baccalauréat et Brevet de technicien (BT), le niveau III aux Brevet de technicien supérieur (BTS) et Diplôme universitaire de technologie (DUT) et les niveaux II et I enfin, aux diplômes de niveau égal ou supérieur à celui des écoles d'ingénieurs ou de la licence.
}

* Philippe Trouvé est professeur au Groupe ESC (École supérieure de commerce)-Clermont et professeur associé à l'université d'Auvergne, où il enseigne et développe des recherches en sociologie et management des ressources humaines. Il est également directeur du Centre associé au Céreq de ClermontFerrand. II fait partie du Centre de recherche clermontois en gestion et management (CRCGM, EA : 38.49) où il dirige l'axe : "Emploi, travail et formes de mobilisation de la main-d'œuvre ». Ses travaux actuels portent sur les restructurations d'entreprises et sur l'évolution des encadrements intermédiaires. 
l'impuissance des dispositifs de formation continue à soutenir l'adaptation et le développement des salariés en place. Un désajustement grandissant s'en serait suivi dans la relation formation-emploi (ou plus exactement «entre les multiples ambitions des politiques de validation - supposées favoriser l'accès à l'emploi - et les carrières professionnelles ascendantes d'une part et la structure professionnelle d'autre part»), illustré par la montée des déclassements à l'embauche. C'est ce constat de déroute collective que P. Santelmann se propose d'éclairer en s'appuyant sur l'examen de la situation des techniciens et agents de maîtrise (TAM) du secteur industriel, qui lui semble emblématique du "décalage entre la politique certificatrice et l'évolution des qualifications réelles ».

La première partie de notre propos s'efforce de présenter et de discuter la thèse centrale de P. Santelmann sur les distorsions grandissantes entre diplômes et qualifications, puis de reprendre d'assez près l'architecture de son argumentaire. Nous choisissons ensuite d'aborder trois points de vue proposés par l'auteur qui nous semblent les plus propices à la discussion. Le premier, qui argumente sur la propension du système éducatif français à répondre systématiquement par l'élévation des qualifications scolaires aux évolutions de l'appareil productif. Celle-ci a été, selon nous, sérieusement renforcée, moins par les pratiques réelles des entreprises que par leur conception déterministe de la modernisation technico-organisationnelle les inclinant pendant longtemps à accroître leur sélectivité par des embauches de substitution en faveur d'un personnel de mieux en mieux et de plus en plus formé. De sorte que, loin d'être l'apanage d'un système éducatif autocentré, la tendance à surestimer l'intellectualisation du travail fut largement partagée par un système productif techno-centré. Par ailleurs, les données statistiques attestent que la montée générale des niveaux de formation ne s'est pas accompagnée d'un «blocage des filières promotionnelles » à l'intérieur des entreprises (deuxième partie); le second point de vue développé par P. Santelmann s'appuie sur l'analyse des TAM pour étayer sa thèse du déclin des apprentissages expérientiels et de l'insuffisante mobilisation de la formation continue dans les processus de professionnalisation des professions intermédiaires. Or cette approche paraît sérieusement battue en brèche du fait du manque de pertinence sociologique de l'agrégat statistique choisi et du niveau d'accès des TAM à la formation continue, bien supérieur à celui des autres catégories professionnelles. Et ceci, même si les dispositifs institutionnels qui visent à valoriser l'expérience ont du mal à se dégager du modèle didactique dominant et à produire une légitimité analogue à celle des titres scolaires. Pour autant, le système éducatif en serait-il plus responsable que les politiques d'entreprises (troisième partie) ? Enfin, le troisième point de vue réaffirmant la suprématie institutionnelle de l'appareil scolaire est relativisé par un rappel sur le rôle du système des relations professionnelles dans la construction des qualifications (quatrième partie).

\section{L'INADÉQUATION ET SES EFFETS DE DÉCLASSEMENT}

Qu'est-ce qui peut donc pousser l'auteur à remettre l'ouvrage sur le métier? Certes, la plupart des travaux de synthèse s'accordent à reconnaître la montée généralisée des niveaux de la formation initiale en même temps que l'accentuation récente de l'inadéquation formation-emploi, tant du point de vue des niveaux que des spécialités. Le phénomène de déclassement à l'embauche par niveaux (normatif et subjectif) concernerait aujourd'hui près de $40 \%$ des jeunes, particulièrement en augmentation chez les titulaires de baccalauréats ou d'un bac +2 , les «désajustements» par spécialité étant en général encore plus prononcés (Couppié, Giret, Lopez, 2005). Pour autant, des évènements nouveaux et suffisamment importants seraient-ils intervenus au cours de ces dernières années, obligeant dès lors à rouvrir le dossier? Sans doute pas. Car en faisant dépendre trop étroitement le constat d'inadéquation des outils de mesure des déclassements, on s'attaque à la fois à la température et au thermomètre. C'est à peu près l'argument développé par Dauty, Lemistre et Vincens (2006, p. 95) : "puisque les diplômes se dévalorisent, les analyses $d u$ déclassement qui reposent sur des normes "institutionnelles" posent problème, non seulement car la mesure normative 
repose sur l'hypothèse d'une stabilité des contenus d'emplois, mais surtout parce que la dévalorisation modifie la correspondance niveau de diplôme-niveau d'emploi d'une génération à l'autre. » Or, en France, ajoutent les auteurs, "la dernière grille de correspondance normative entre emploi et formation repose toujours sur la nomenclature de $69 »$. Si bien que, faute de révision de la Nomenclature des niveaux de formation (NNF), il faudrait s'en tenir à l'hypothèse plausible d'un déplacement actuel des normes de qualification (Fournié, Guitton, 2008) ${ }^{2}$ qui, sans donner entière satisfaction aux acteurs d'une relation formation-emploi dégradée (et surtout pas à ceux qui sont déclassés !) n'en atténuerait pas moins le caractère dramatique.

D'ailleurs, n'est-ce pas déjà une réalité dans la perception subjective des acteurs qui ont intériorisé les nouvelles normes (Giret, Lemistre, 2005; Moncel, 2008) ? À commencer par les employeurs pour lesquels le diplôme fonctionne toujours comme un « signal » ou un « filtre » et non comme un équivalent intangible de l'emploi ? C'est ce qui expliquerait - comme le montrent Giret et Hatot (2001, p. 64) en s'appuyant sur l'étude du déclassement à l'embauche des DUT et BTS - qu'en dépit de l'existence du système de classification et des conventions collectives qui devraient inciter l'employeur à payer le jeune en fonction du niveau de qualification de l'emploi, «pour un poste d'employé ou d'ouvrier, le salaire du premier emploi des diplômés de BTS ou de DUT est en moyenne de $15 \%$ supérieur à celui des jeunes sortants de niveau bac» [ce qui montre que si] « les employeurs ne proposent pas à leurs salariés un emploi correspondant à leur formation, ils rétribuent partiellement leur diplôme supplémentaire».

Au final, il n'est pas facile de trancher (mais faut-il trancher ?) en isolant les effets du progrès technique ou des transformations organisationnelles et ceux liés à l'inflation relative de diplômes qui viennent biaiser la mesure des déclassements : «La relation formation-

\footnotetext{
${ }^{2}$ C'est ainsi que, pour les seules professions intermédiaires de l'industrie - où l'on a toutes les chances de retrouver les techniciens et les agents de maîtrise, tandis que la norme de qualification des 50 ans ou plus (entendue comme le niveau de diplôme le plus fréquent pour une catégorie d'emploi donnée) se situe aux niveaux V et VI pour $73 \%$ d'entre eux -, elle s'établit aujourd'hui aux niveaux III et I-II pour $62 \%$ des moins de 30 ans.
}

emploi pouvant être qualifiée de "normale » à un instant $t$ n'est pas stable dans le temps, ce qui rend difficile la quantification du phénomène de déclassement à l'embauche, en particulier pour les jeunes titulaires au plus d'un niveau IV [...] les compétences requises pour occuper un emploi dont la qualification n'a pas changé, en termes de catégorie socioprofessionnelle, s'accroissent, d'où une évolution de l'emploi non reconnue dans les grilles de classification.»(Blum 2003, p. 46)

C'est pourquoi, délaissant ce déjà volumineux dossier " adéquationniste », nous nous concentrerons plutôt sur ce qui nous paraît être l'apport et le mérite de P. Santelmann : sa recherche d'explications historiques et plus encore son analyse interprétative des effets multiples du désajustement formation-emploi décrit plus haut. Ce faisant, nous suivrons attentivement le mouvement du texte tout en en dégageant les hypothèses qui nous paraissent les plus stimulantes.

L'argumentaire général comporte quatre volets successifs avant de converger vers une conclusion très riche qui mêle l'illustration des lacunes du système éducatif (excès de spécialisation et de cloisonnements, offres de formation illisibles ou contradictoires, exclusion des non-diplômés, etc.) - moins pris en lui-même que comme produit d'un modèle social - et la défense des savoirs expérientiels ou de la réactivation des compétences à travers la formation continue, bien souvent négligée dans notre modèle national. Il plaide alors pour une introduction plus ferme des cultures technologiques et professionnelles dans le système scolaire (à l'instar du modèle allemand) et pour une réintroduction des ambitions de la formation professionnelle dans le champ du dialogue social.

La première partie est consacrée au décryptage de l'activité fonctionnelle des TAM. Après avoir montré la dispersion intersectorielle, la prédominance des activités de production et le caractère "brouillé » et poreux du niveau d'emploi concerné par cette catégorie socioprofessionnelle prise comme un tout, l'auteur se centre sur le travail industriel et tente d'en résumer les grandes transformations (deuxième partie). Outre les innovations technologiques, ces transformations sont marquées essentiellement par des réorganisations qui élargissent le travail ouvrier 
et "troublent son identification", sans doute aux deux sens du terme, c'est-à-dire d'identité professionnelle et de codification des emplois. Du point de vue des TAM, deux mouvements contradictoires semblent se dessiner. Les techniciens verraient leur effectif s'accroître grâce à leur rôle central dans la diffusion et la maintenance des nouvelles technologies dans tous les univers du travail, lui-même basé sur le déploiement de nombreuses fonctions d'intervention et l'industrialisation croissante des activités (y compris tertiaires). Les encadrements intermédiaires, de leur côté, subiraient un inexorable déclin en grande partie dû au raccourcissement des lignes hiérarchiques. Selon l'auteur, en dépit de nombreuses difficultés liées tout à la fois à l'absence de profils adéquats en provenance de l'enseignement et à l'insuffisance de formation continue pour répondre aux nouvelles exigences de flexibilité, de qualité et de fiabilité, ce sont "les personnels en place » qui ont mené la plupart du temps ces changements, aidés en cela par la persistance d'espaces promotionnels dans les entreprises entre les catégories d'ouvriers non qualifiés et qualifiés et entre les ouvriers qualifiés et les fonctions de technicien. Un tel phénomène vertueux d'acquisition expérientielle de compétences aurait surtout joué, selon P. Santelmann, au début des années 80 et jusqu'au début des années 90, dans la période qui a précédé probablement un afflux plus important de jeunes diplômés sur le marché du travail.

La troisième partie révèle une rupture par rapport à ce qui vient d'être dit. Brusquement, au détour des années 90, les entreprises, renonçant « à associer les salariés en place aux modernisations» auraient commencé à embaucher des jeunes "surdiplômés» considérés comme «plus dociles et moins rémunérés », notamment des BTS et des DUT, la politique des offreurs de travail s'alimentant ici à la massification des enseignements secondaire et supérieur. Pour P. Santelmann, une telle orientation aurait eu pour effet de délaisser l'effort de formation promotionnelle envers le personnel en place, de déprécier les formations ouvrières, d'exclure progressivement $\mathrm{du}$ monde industriel les moins diplômés de l'enseignement professionnel, tout en favorisant, selon le mot d'A. d'Iribarne, cité par Santelmann, une sorte d' 'enfermement catégoriel pour les diplômés de niveau III ». P. Santelmann a le mérite ici de noter que, dans la réalité, "les incidences des nouvelles organisations du travail, en termes de niveaux de qualification » n'ont pas été aussi radicales que prévu, que la reconfiguration des organisations n'a pas été aussi profonde qu'annoncée et, citant E. Quenson, que derrière les rhétoriques managériales, la structure des qualifications «reste bien souvent inchangée ».

Une conclusion immédiate devrait en être tirée : celle de l'inanité d'une analyse préalable des «besoins futurs » de l'industrie pour agir sur la structure des diplômes, car l'une des leçons de la dernière décennie est que les entreprises sont aujourd'hui de moins en moins capables de faire de la prospective quantitative et qualitative des emplois un outil de gestion suffisamment fiable. À plus forte raison une prospective du travail et des compétences ${ }^{3}$ dont l'auteur persiste à penser indirectement qu'elle irait de soi, jusque dans les PME: «les difficultés d'ajustement entre le système de formation et les organisations du travail n'ont toujours pas été résolues et les entreprises industrielles (notamment les PME) sont confrontées à une pénurie récurrente de compétences adaptées aux nouveaux registres d'activité et aux organisations nouvelles. »

Abordant la période la plus récente, la quatrième partie enfin met l'accent sur les désajustements du système de formation. Sur ce point, la thèse défendue par l'auteur s'écarte sensiblement de la perception commune: avant de structurer très fortement l'ensemble du système des qualifications à la française, le modèle scolaire aurait été lui-même contaminé par un principe de hiérarchisation taylorien marqué notamment par un clivage quasi infranchissable entre fonctions de conception et d'exécution dans le travail et formation générale ou technique et formation professionnelle dans l'appareil éducatif. Cette sorte de bouclage, qui serait notre caractéristique nationale, aurait non seulement résisté à la poussée de nouvelles formes d'organisation du travail, désormais moins cloisonnées et plus interactives, mais aussi phagocyté le développement de l'apprentissage et de l'alternance où «les phases d'apprentissage en situation de travail ont insuffi-

3 P. Ughetto (2007) s'interroge d'un point de vue managérial:
"sait-on gérer la montée des exigences?" 
samment modifié le contenu des formations dispensées dans les lieux d'enseignement», jusqu'aux certifications de branche (les CQP : Certificats de qualification professionnelle). Retour donc à la case départ d'un véritable modèle sociétal dont $\mathrm{E}$. Verdier (1995, 1996, 2001) a maintes fois démonté la cohérence et donc, la résistance. Modèle auquel n'échappent pas les DUT-BTS, incapables, selon l'auteur, d'incorporer sous la forme scolaire une grande partie des compétences relationnelles, d'autorité ou d'expertise, requises par les nouvelles situations de travail et dont l'apprentissage relèverait tout autant d'accumulations expérientielles. De plus, en maintenant le clivage culturel entre les trois filières de formation (professionnelle, technique et générale), ces formations supérieures courtes à visée professionnalisante, tout comme celles des niveaux IV, auraient échoué à faire émerger de véritables filières promotionnelles pour les ouvriers de production issus de l'enseignement professionnel.

Nous l'avons dit plus haut, plusieurs lignes d'interprétation structurent le propos de P. Santelmann, qui sont autant de thèses ou d'hypothèses stimulantes, et donc à commenter ou à discuter. Nous le ferons en mettant en évidence trois grands pôles de questionnements : l'un a trait aux relations entre l'afflux des diplômés et le devenir des voies promotionnelles; l'autre porte sur la pertinence de la population statistiquement agrégée utilisée par P. Santelmann pour asseoir sa démonstration ; enfin, nous nous demanderons si une incursion dans le domaine des relations industrielles ne serait pas propice à un renouvellement du débat engagé par l'auteur.

\section{LA COEXISTENCE DE L'AFFLUX DES DIPLÔMÉS ET DES VOIES PROMOTIONNELLES}

La première thèse concerne la signification de la brusque élévation du niveau de formation initiale, au détour de la décennie 90. Au-delà de la tradition nationale et des intérêts conjugués de l'ensemble des acteurs, P. Santelmann l'attribue à une interprétation erronée des mutations techniques et organisation- nelles de l'époque, perçues « dans la sphère éducative (nous soulignons) comme un mouvement d'intellectualisation et d'abstraction du travail qui venait en quelque sorte justifier, a posteriori, la distinction classique entre savoirs généraux et technologiques et savoirs professionnels ». Cette thèse de l'intellectualisation du travail, y compris de la dématérialisation du travail ouvrier ou de la «matérialisation complète de l'intelligence [dans la] machine» (Kern, Schumann, 1989, p. 172)4, ne fait pas de doute.

Mais, à y regarder de plus près, elle ne fût pas l'apanage des acteurs du système éducatif. Elle fût tout aussi répandue, notamment tout au long des années 80 , dans une fraction du patronat modernisateur qui tenait alors un discours de rupture sur les nouveaux profils professionnels exigés. D'ailleurs, l'apparition des bacs pros en 1985 ne correspondaitelle pas à ces nouveaux besoins? Dans les milieux de la recherche, les positions adoptées sur cette question furent infiniment plus prudentes que celle de Gambier et Vernières prédisant la « réduction du rôle de l'apprentissage par l'expérience» (1991, p. 173) : soit qu'elles n'aient pas tiré toutes les conclusions de la « requalification » sur la restructuration des formations ouvrières (Kern et Schumann et leur fameuse Fin de la division du travail, 1984), soit qu'elles aient assorti le mouvement d'abstraction et de complexification du travail d'une grande diversité de modes de gestion dans les entreprises en relativisant le déterminisme technico-organisationnel (Coriat, 1990, notamment chap. $\mathrm{V}^{5}$, mais aussi Maurice et al., 1985). Ce sont précisément ce mouvement et la dénonciation concomitante d'un manque de qualification chronique de la maîtrise industrielle traditionnelle qui firent croire, pendant longtemps, à son déclin inexorable, voire à sa disparition prochaine (Trouvé, 1996-a). Sur ce point, la prévision du

\footnotetext{
${ }^{4}$ Il est singulier de constater que, durant les années 80 , la thèse de l'intellectualisation du travail (des plus qualifiés) se soit souvent combinée avec le mythe de "l'ouvrier mécatronique », c'est-àdire des robots en séries ou de « l'usine sans hommes ».

5 Tout en relevant l'émergence de la nouvelle figure professionnelle des «ouvriers-opérateurs de conduite de systèmes automatisés », B. Coriat ne la magnifie pas. La montée de «l'abstraction» par « dissipation du travail direct », « extension du travail indirect » et « interpénétration » des deux, se traduit dans les faits par des solutions très diverses, suivant les traditions et les cultures d'entreprises, la nature de leur positionnement sur les marchés ou les produits... » (1990, p. 219).
} 
Commissariat général du plan - encore en 1999 («Le rôle des agents de maîtrise est amené à se réduire et à se transformer, sinon à disparaître »), rappelée par P. Santelmann, ne s'est pas réalisée. Non seulement cette catégorie professionnelle résiste mieux que l'on ne l'avait prédit (après un déclin progressif de ses effectifs jusqu'en 2000, compte tenu de la désindustrialisation, elle est repartie depuis à la hausse, retrouvant un indice proche de celui du milieu des années 80), mais les niveaux de diplôme VI $(22 \%)$ et V (45 \%) y sont encore bien présents en 2005. Ce qui voudrait dire que, contrairement à ce que pense l'auteur, le recrutement important de jeunes BTS-DUT (niveau III) sur des emplois d'agents de maîtrise (actuellement $14 \%$ pour la seule industrie et tous secteurs confondus) n'a pas fondamentalement «bloqué les filières promotionnelles ». Les modes d'accès externes et verticaux coexistent donc bel et bien pour cette catégorie intermédiaire un peu trop rapidement évacuée. Et il en est de même pour la catégorie techniciens, où la proportion de débutants diplômés bac +2 n'a que faiblement progressé depuis 20 ans $(40 \%$ en 1985, $50 \%$ en $2005)$ et où les niveaux $\mathrm{V}(26 \%$ et même $31 \%$ pour la seule industrie) et VI $(14 \%)^{6}$ sont encore très fortement représentés.

En outre, en exploitant l'Enquête FQP 2003, M. Möbus (2009) établit que si $22 \%$ des salariés classés agents de maîtrise ont débuté dans la vie active dans cette catégorie, ils étaient $65 \%$ à avoir débuté comme ouvriers ; de même, si $43 \%$ des techniciens ont débuté comme techniciens, ils étaient $46 \%$ à avoir été d'abord ouvriers dont la promotion correspond le plus souvent à un «reclassement». Une preuve que les filières promotionnelles chez les TAM sont loin d'être asséchées par l'afflux de jeunes plus diplômés en provenance du marché externe.

Selon M. Möbus, l'absence de répercussion des « recrutements de débutants issus de la voie technologique (BTS, DUT) sur le profil actuel de l'ensemble de la catégorie "s'expliquerait par les flux de sortie précoces de la catégorie puisqu'en 2003 , environ $35 \%$ et $32 \%$ des salariés qui ont débuté respectivement techniciens et agents de maîtrise

${ }^{6}$ L'ensemble de ces données provient d'une exploitation de l'Enquête Emploi de l'INSEE. sont devenus cadres. Les raisons de la bonne résistance des filières promotionnelles et du maintien d'une main-d'œuvre intermédiaire traditionnelle ont été longuement abordées pour les contremaîtres d'industrie (Trouvé, 1997). L'une d'entre elles, évoquée incidemment par P. Santelmann, est à notre avis à prendre très au sérieux : la transformation des organisations de travail n'aurait finalement pas été aussi poussée et surtout son impact sur les structures de qualification n'aurait pas été aussi déterminant que prévu. Une réflexion qui vaut surtout pour la décennie 2000 et qui confirmerait la périodisation défendue par l'auteur. Car les grandes mutations technologiques (informatisation et restructuration des lignes de production) et organisationnelles (diffusion du juste-à-temps, raccourcissement des lignes hiérarchiques, décentralisation de la gestion, démarches qualité) qui ont joué sur la recomposition de la main-d'œuvre (en faveur de la plus qualifiée) sont désormais derrière nous, la décennie actuelle ayant été surtout occupée par le gigantesque jeu de lego mondial de la fragmentation (décompositionrecomposition) des chaînes de valeur (Berger, 2006) et notamment de la supply chain $^{7}$, un terrain qui ne s'est sans doute pas traduit par une déformation de la structure des emplois et des qualifications aussi nette que dans les vingt années précédentes.

\section{LA PERTINENCE D'UN AGRÉGAT STATISTIQUE}

Au sortir d'une telle série de données, on finirait par se demander si l'agrégat statistique constitué par les TAM est tout à fait pertinent pour illustrer deux thèses particulièrement insistantes dans le texte de P. Santelmann : celle de la sous-estimation grandissante des apprentissages expérientiels et celle de la mobilisation insuffisante de la formation continue.

\footnotetext{
${ }^{7}$ La supply chain désigne l'ensemble des opérations et procédures qui permettent de gérer de façon optimale la totalité des flux physiques, des informations et des interfaces entre les différents acteurs, producteurs et fournisseurs qu'implique la fabrication d'un produit ou d'un service. La traduction française de « chaîne logistique » est de ce fait nettement insuffisante car elle laisse de côté les flux d'informations concomitants. La supply chain pourrait s'assimiler parfois à la chaîne de valeur dans la mesure où, à travers elle, les organisations cherchent à se procurer un avantage concurrentiel.
} 
Nous insisterons dans un premier temps sur l'hétérogénéité sociologique de la population étudiée par l'auteur. Puis, nous montrerons que son accès à la formation continue n'est pas négligeable. Enfin, nous nous interrogerons sur la place ambivalente de l'expérience dans les processus de professionnalisation des catégories intermédiaires, moins du point de vue de la gestion des entreprises où son rôle semble acquis (voir plus haut), que des dispositifs institutionnels visant à la revaloriser (apprentissage, Certificats de qualification professionnelle, Validation des acquis de l'expérience...). Il en ressort un tableau nuancé, attestant d'une période transitoire, en tout cas moins tranché que celui proposé par P. Santelmann, sur les difficultés des apprentissages expérientiels à se faire reconnaître.

Car, on vient de le voir, les trajectoires de professionnalisation des fractions les plus anciennes de la catégorie recrutées sur le marché interne, comme des débutants issus des filières technologiques supérieures, dépendent sans doute ici plus qu'ailleurs des pratiques de gestion des ressources humaines des entreprises. D'où une très forte hétérogénéité des niveaux de recrutement, des profils et des emplois, peu propice à des généralisations, non seulement inter-catégorielles (car, comme nous le verrons plus loin, l'association statistique des techniciens et agents de maîtrise constitue en bien des points une anomalie sociologique), mais également intracatégorielles. Pour les techniciens par exemple, en utilisant l'Enquête FQP, M. Möbus montre bien la grande diversité de fonctions occupées et de contenus d'activité entre les deux filières d'accès à la catégorie (externe et interne), tandis que «les fonctions recherchelétudes/méthodes sont le plus souvent citées par les débutants [...] pour les promus, les fonctions installation/maintenance et production/ exploitation arrivent en tête » (2009, p. 42).

Par ailleurs, techniciens et agents de maîtrise se distinguent par leur répartition selon les secteurs d'activité (les premiers sont plus nombreux dans l'industrie et les services, les seconds dans la construction), les métiers pratiqués, leur niveau de diplôme ou leur âge moyen (tandis qu'un quart des techniciens sont jeunes, la proportion des moins de 30 ans ne dépasse pas $10 \%$ chez les agents de maîtrise $)^{8}$. De plus, les conditions vécues de l'exer- cice de leur activité ne sont pas non plus les mêmes (Enquête \& Opinion/UGICT-CGT, 2006). Par exemple, alors que $23 \%$ des agents de maîtrise considèrent que «leur métier, leur activité professionnelle n'évolue pas» (ce qui en dit long sur l'effectivité des mutations managériales), ils ne sont que $11 \%$ des techniciens à avoir la même perception. Tandis que cette évolution est due pour $74 \%$ des seconds aux évolutions scientifiques et techniques, elle est prioritairement attribuée par les premiers aux «objectifs de production» (45\%) aux «politiques clients ou usagers » $(50 \%)$ et aux «contraintes de temps» $(40 \%)$. Alors que les agents de maîtrise semblent avoir davantage le sentiment d'appartenance à un collectif de travail que les techniciens (64\% contre $57 \%$ ), ils sont $48 \%$ à estimer que "l'exercice de leur métier interfère négativement sur leur vie privée» contre $21 \%$ des techniciens. Tandis que $53 \%$ des techniciens ont le sentiment que leur autonomie a augmenté au cours des dernières années, ils ne sont que $39 \%$ des agents de maîtrise à avoir le même sentiment. Pour les responsabilités, les proportions s'inversent, avec un sentiment plus fort (59\%) chez les agents de maîtrise que chez les techniciens (52\%). À égalité (57\%), les deux catégories estiment pouvoir connaître une progression de carrière, ce qui rejoindrait les données objectives présentées plus haut. À l'inverse, c'est la "reconnaissance sociale» et «le soutien de la hiérarchie» qui opposent les deux catégories: alors que les techniciens estiment majoritairement en bénéficier, les agents de maîtrise prétendent en être privés.

En outre, en matière de formation continue à l'initiative de l'entreprise, on sait que ce sont les professions intermédiaires et plus particulièrement les TAM qui, depuis plus de 20 ans, ont, avec les cadres, le taux d'accès le plus élevé et bénéficient de l'effort quantitatif le plus important, soit de l'ordre de 18 heures de formation par an et par salarié de la catégorie, contre 12 heures par salarié pour l'ensemble des catégories. Une partie au moins de cet avantage pourrait s'expliquer par la faible articulation a priori entre la formation initiale (et ses titres) et la diversité des emplois occupés, rendant nécessaires - au-delà de compétences transversales -

${ }^{8}$ L'établissement de ces données chiffrées doit beaucoup à M. Möbus (2009). 
les ajustements au cœur de métier des entreprises (pour les techniciens) ou aux nouvelles technologies managériales pour favoriser un engagement, une responsabilité et une autonomie plus grandes des collectifs de travail (pour les agents de maîtrise). Il est vrai que sur ce point, une exploitation plus poussée des enquêtes sur la Formation continue du Céreq serait aujourd'hui indispensable sur les contenus de la formation, non seulement formelle mais aussi informelle en situation de travail, pas seulement institutionnalisée mais certifiante. Il existe en tout cas, pour les TAM comme pour les autres catégories, une corrélation forte entre formation professionnelle continue et mobilité interne. La spécificité des TAM tiendrait à ce que l'une et l'autre se situent à un niveau proche de celui des cadres et que les caractéristiques du poste ou de la fonction occupée sont ici plus qu'ailleurs "plus discriminantes que celles de la personne pour les opportunités de formation continue et de changement de poste dans l'entreprise » (Marion, Möbus, Sigot, 2008, p. 14).

S'agissant des TAM, la progression de l'apprentissage et de l'alternance au cours de ces dernières années, dans l'enseignement supérieur, pourrait constituer un atout supplémentaire dans leur professionnalisation. Pour les seuls niveaux III, qui ne représentent encore que $12 \%$ de l'effectif total des apprentis, cette modalité d'acquisition de compétences théoriques et pratiques a en effet connu une croissance de $32 \%$ (Demongeot, Leprévost, 2008). Mais il est vrai qu' « au fur et à mesure que s'élève le niveau de formation, les domaines professionnels des services prennent le dessus sur les domaines de la production. Encore majoritaires au niveau $V$ (71,5\%), les spécialités de production ne représentent plus que $38 \%$ des effectifs d'apprentis au niveau III et 18,5\% au niveau II » (idem). D'où les dangers d'une "scolarisation rampante» (Moreau, 2008) de cette voie de formation dont P. Santelmann souligne non sans raison l'impuissance à transformer le contenu des diplômes professionnels ou à élargir le "périmètre d'évaluation et de certification des savoirs ainsi construits ». Par ailleurs, cette progression de l'apprentissage dans l'enseignement supérieur n'a ni entrainé une revalorisation de l'enseignement professionnel des plus bas niveaux de formation, ni bouleversé les correspondances entre la hiérarchie des formations et les origines sociologiques des jeunes apprentis: avec l'élévation des niveaux de formation, on assiste en effet à une éviction progressive de ceux qui sont issus des classes populaires. En s'appuyant sur une enquête conduite dans les Pays de la Loire, G. Moreau souligne cet effet d' «éclatement social [actuel] de l'apprentissage» : «la proportion de pères ouvriers est de $64 \%$ en CAP, de $56 \%$ en $B E P$, de $52 \%$ au niveau $I V$, de $38 \%$ en BTS et de $27 \%$ pour les autres apprentis du supérieur. La part des enfants de cadres et de professions intermédiaires suit le chemin inverse, avec respectivement $11 \%, 15 \%, 18 \%, 30 \%$ et $37 \%$. Si l'apprentissage [...] ouvre parfois le chemin de la mobilité scolaire [...] la tendance lourde du dispositif reste l'élimination progressive du milieu populaire au fur et à mesure de l'élévation du niveau de formation.» (2008, p. 125)

Quant aux Certificats de qualification professionnelle (CQP) délivrés par les branches professionnelles, permettant aux salariés «d'acquérir une qualification opérationnelle reconnue», l'auteur a raison de soupçonner qu'ils sont venus renforcer la logique de spécialisation et de prolifération des titres sur un marché du travail qui n'en aurait pas demandé autant. Ils se sont en effet tellement multipliés au cours de ces dernières années, qu'il y en aurait aujourd'hui plus de 400 contre environ 220 CAP : un bel exemple d'«illisibilité », généralement reproché au système éducatif! Bien plus, si les entreprises ont tendance à davantage utiliser les CQP pour les ouvriers que pour les techniciens ou agents de maîtrise, n'est-ce pas que la prétendue inadéquation des formations scolaires est moins importante pour les BTS et DUT que pour les CAP et BEP industriels (Veneau, Personnaz, 2002)?

En dépit de l'absence de recul, le vaste chantier de la Validation des acquis de l'expérience (VAE) aurait, selon nous, mérité un meilleur sort. Les premiers travaux d'observation sur les dispositifs et les modes d'usage de cette nouvelle forme de certification professionnelle, instaurée en 2002, montrent certes que ce sont à nouveau les cadres et les professions intermédiaires qui en sont les principaux bénéficiaires dans l'enseignement supérieur et que le cadrage institutionnel $\mathrm{y}$ est par définition très 
prégnant $(76 \%$ des dossiers traités par l'Éducation nationale, selon Le Roux, 2005).

Mais ces dispositifs, sans doute encore très tâtonnants, n'organisent-ils pas déjà des ponts et même des modalités de correspondance entre savoirs pratiques et savoirs théoriques qui échapperaient tout à la fois à la rigidité et à l'exclusivité des normes scolaires ainsi qu'au pouvoir discrétionnaire des employeurs pour qualifier le travail ? Plutôt que de vouloir à tout prix professionnaliser l'enseignement, l'opportunité ne se présenterait-elle pas ici de certifier à rebours l'expérience acquise ou tout au moins de faire fonctionner simultanément les deux mouvements? Deux signaux nous semblent ici dignes d'être relevés et générateurs d'investigations pour l'avenir: d'une part, en s'appuyant sur l'examen de la VAE dédiée à l'enseignement supérieur, Le Roux (2005) remarque que "plus les bénéficiaires sont jeunes plus ils valident des diplomes professionalisants (licence professionnelle, DUT, IUP - Institut universitaire professionnalisé...) alors que les 46 ans et plus tentent de faire reconnaître leur expérience acquise par des diplômes généraux (licence, maîtrise, DEA-DESS - diplôme d'études approfondies et diplôme d'études supérieures spécialisées) »; d'autre part, d'un point de vue plus général, Ch. Labruyère (2006) semble ouvrir une brèche dans la tendance à la montée inexorable des titres scolaires: "globalement, en 2004, près de quatre candidats à la VAE sur dix tentaient d'obtenir un diplôme de niveau $V$, près d'un quart visaient un niveau IV, un autre quart un diplôme de niveau $b a c+2$, un sur dix un bac +3 au minimum $[\ldots]$ $37 \%$ des dossiers de VAE ciblant un bac +2 sont traités par l'EN [...] la plus grosse part, validée par les rectorats (42\% pour les BTS) et $17 \%$ seulement par l'enseignement supérieur (DUT). » Ce qui voudrait dire que la formation expérientielle (sauf à restreindre l'expérience à l'acquisition de qualifications spécifiques), ne suffit pas en ellemême à valider socialement les qualifications. De sorte que pour les acteurs qui en sont porteurs, si le diplôme comme «validation sociale d'acquis scolaire [...] ne vaut pas reconnaissance professionnelle par le système productif $»(\mathrm{P}$. Santelmann citant Lochet), l'expérience professionnelle proposée notamment aux jeunes débutants de niveau III - dont on a vu qu'elle est marquée par le déclassement à l'embauche - ne suffirait pas à apaiser leur impatience à valider intégralement leur qualification. C'est l'occasion de passer à un troisième ensemble de questions suggérées par le texte de P. Santelmannn.

\section{LE RÔLE DE LA RÉGULATION SALARIALE DANS LA PRODUCTION DES RIGIDITÉS}

À trop vouloir mettre en scène la prédominance du modèle scolaire, centralisé, rigide et administratif de la qualification, dans l'établissement des systèmes de formation et d'emploi, ne finirait-on pas par oublier son caractère de construit social et le jeu des acteurs qui l'ont produit, seuls capables d'expliquer sa stabilité et sa durabilité dans le temps ? En rappelant les origines planificatrices de la Nomenclature des niveaux de formation (NNF), Dauty, Lemistre et Vincens (2006, p. 11) situent bien le problème : «Il ne s'agi[ssai] t pas de décrire une situation existante mais d'élaborer une norme souhaitable des relations entre niveaux de formation et niveaux d'emploi pour prévoir le développement de la formation (éducation). »

De fait, comme le soutient P. Santelmannn, c'est cette norme collective qui s'est progressivement imposée, pour gérer les filières et les niveaux de certification, mais aussi et surtout, dans tout le champ social, des politiques du travail ou de la statistique publique aux critères de recrutement des entreprises, en passant par la structuration des grilles de classification dans les conventions collectives à l'échelle des branches. Mais cette prééminence du modèle scolaire dans le système des relations formation-emploi n'oblige en rien à négliger les autres acteurs et notamment les employeurs qui, à l'échelle de la branche ou de l'entreprise, ont largement contribué à entretenir la norme collective. De sorte qu'il s'agirait moins de savoir comment celle-ci a progressivement triomphé, mais comment elle révèle un compromis, une sorte de "pacte social » (Trouvé, 1996-b) qui confère cohérence et endurance au système. Sinon comment concevoir que celle-ci ait pu résister aux vagues successives des mutations du 
travail ? Pour ce faire, il conviendrait alors de resituer la relation formation-emploi dans la dynamique des relations professionnelles et de concevoir la qualification, non comme le produit d'une institution (étatique) toute puissante ou de pures règles de marché, mais d'une activité de régulation entre des acteurs aux stratégies multiples.

Les articulations profondes que l'auteur déplore entre l'espace des qualifications et le système des classifications nous semblent, de ce point de vue, très éclairantes. Ces classifications seraient-elles aujourd'hui trop rigides ou trop attachées aux diplômes pour faire front aux besoins de flexibilité et de polyvalence? Elles sont en tout cas bel et bien le produit d'une négociation collective qu'il conviendrait de réintégrer comme une dimension clef de toute analyse de la relation formation-emploi et de ses dysfonctionnements. On comprendrait alors mieux les enchaînements historiques qui ont conduit les partenaires sociaux des grilles dites Parodi au «modèle de la compétence » (Zarifian, 1999, 2001), sans que le socle de la référence à la hiérarchie traditionnelle des emplois n'ait été vraiment remis en cause. Car il s'agit d'un point de stabilité majeur, à forte légitimité collective, dans la régulation salariale (Saglio, 1988). C'est ce qui expliquerait qu'en dépit d'une forte inflexion engagée depuis plusieurs décennies vers une conception plus individualisée de la qualification (critères classants, puis logique compétence à partir de l'accord historique A Cap 2000), qui pouvait laisser craindre un déplacement du «pouvoir de qualifier le travail dans les mains des seuls employeurs » (Coutrot, 1999, p. 39) c'est-à-dire hors du champ traditionnel de la régulation collective de branche, les partenaires sociaux ne se sont pas précipités pour signer des accords dans le domaine, encore moins des accords liant très explicitement formation continue, évolution de carrière et classification. D'où leur nombre encore très limité aboutissant à l'introduction complète de la logique compétence (Tallard, 2001, p. 170); d'où la multiplication des panachages entre formules mixtes (y compris "grille Parodi rénovée »), l'adoption même de critères classants étant loin d'être généralisée (Dauty, Lemistre, Vincens, 2006, p. 50). Sans compter les débats multiples sur les effets ou non de rupture de la logique compétence (Oiry, d'Iribarne, 2001) et les acrobaties théoriques pour suggérer un nouveau compromis social autour d'un «cercle vertueux qualification-compétence », la première constituant un «potentiel» sur lequel viendrait s'ancrer la seconde (Paradeise, Lichtenberger, 2001). Une chose est sûre, nous ne trouvons pas trace, dans la réalité actuelle, d'un "nouvel échange salarial» ( employabilité contre performance») annoncé par J.-D. Reynaud (2001), qui viendrait sinon se substituer, du moins enrichir l'usage persistant de grilles standardisées de classification des emplois. Pour deux bonnes raisons: d'une part, les éventuelles politiques d'entreprises orientées vers l'employabilité de leurs salariés sont encore dans les limbes; d'autre part, on a occulté le fait que l'évaluation individualisée des compétences acquises s'accompagne de coûts de gestion non négligeables (formation de l'encadrement, préparation et réalisation des entretiens de carrière...) (Ughetto, 2008). C'est ce qui expliquerait, selon nous, que la plupart des employeurs font actuellement un usage sélectif de la gestion des compétences, qui consisterait moins à saisir la contribution des individus qu'à contrôler leur productivité, tout en évitant de mettre trop radicalement en cause une hiérarchie des emplois qui assure depuis bien longtemps la stabilité de l'édifice des relations professionnelles. Et d'ailleurs, un décrochage trop brusque des normes collectives patiemment et historiquement construites, ne risquerait-il pas d'accentuer le brouillage des " qualifications requises », tout aussi préjudiciable à la relation formation-emploi que l'opacité des diplômes et des spécialisations généralement attribuée à la sphère éducative ?

Pour conclure, la contribution de P. Santelmann suscite des sentiments mêlés chez le lecteur. Un sentiment abrupt d'agacement tout d'abord, tant la thèse principale soutenue apparaît réductrice, univoque et proche de la perception commune. Oubliés, le rationnement durable des emplois et la place spécifiquement occupée par les jeunes sur le marché du travail ; oubliés la sélectivité des entreprises et le chômage "d'insertion» ou de «file d'attente» (Verdier, 1995) qui incitent les jeunes à se maintenir le plus longtemps possible dans le système éducatif: les distorsions diplômes-qualifications, selon P. Santelmann, seraient essentiellement dues à la surproduction de diplômés, elle-même consé- 
quence d'une emprise excessive des normes scolaires sur l'ensemble de la relation formation-emploi.

Mais un sentiment de gratitude aussi, en ce que l'intervention de P. Santelmann, au cœur d'un débat qui n'a jamais cessé d'agiter le Céreq, incite à revisiter à la lumière de travaux récents ou anciens, une série de questions habituellement traitées de façon séparée. Il en ressort quelques points forts qui pourraient être autant de balises sur des recherches antécédentes ou subséquentes.

De façon générale, on ne saurait traiter des dysfonctionnements de la relation formation-emploi sans mettre en évidence des processus de co-construction et de régulation collectives qui n'engagent pas seulement le système éducatif, certes prédominant dans la configuration française, mais aussi les entreprises, les acteurs d'intermédiation du marché du travail, les stratégies individuelles ou sociologiquement déterminées des salariés déjà en place ou en phase d'insertion ainsi que le fonctionnement du système des relations professionnelles. Nous avons montré que l'articulation entre les classifications et les normes de niveaux de formation est une illustration de ce travail de régulation collective. En se dégageant d'une analyse disjonctive qui attribuerait trop exclusivement la responsabilité des grippages à l'un ou l'autre des acteurs, une vision plus nuancée apparaît désormais. C'est ainsi par exemple qu'une grande partie des « distorsions » diplômes-qualifications relèvent tout autant d'un déplacement des normes sociales de qualification que de l'emprise d'un système scolaire autocentré. La mesure du déclassement qui pourrait en être la traduction s'avère de ce fait plus difficile à manier que prévu.
De même, la propension française à confondre la modernisation productive avec l'intellectualisation des activités de travail ne saurait être conçue comme le pur produit des logiques éducatives. De ce point de vue, la " surproduction de diplômes » a répondu tout autant aux tensions du marché du travail et à la «montée des exigences» de l'appareil productif, porteur d'une conception technocentrée de la modernisation. D'où l'essoufflement du recrutement des CAP-BEP et le relatif « succès » des bacs pros ou des formations supérieures courtes.

Également, contrairement aux craintes de P. Santelmann, les données statistiques les plus récentes montrent que l'afflux croissant de diplômés sur le marché du travail n'a pas «bloqué les filières promotionnelles ». Les deux voies d'accès aux emplois coexisteraient bel et bien, notamment dans les professions intermédiaires qui constituent souvent une nébuleuse brouillée, non seulement par la multiplicité des contenus d'activité qu'elles recouvrent, mais aussi par «l'instabilité des choix patronaux » à leur égard (Bouffartigue, 1994). C'est ce qui expliquerait que le constat de déclin des savoirs expérientiels dans les processus de professionnalisation soit loin d'être généralisable, pas plus d'ailleurs que celui de l'insuffisante mobilisation de la formation continue. Il est vrai qu'une contribution élevée des entreprises dans la formation de leurs salariés ne se traduit pas forcément par un retour sur investissement pour les employeurs et par des qualifications assimilables à celles qui ont été acquises au cours de la formation initiale pour les salariés. C'est ce qui ressortirait par exemple d'une analyse de la situation particulière des agents de maîtrise du secteur industriel (Trouvé, 2009). 
Berger S. (2006), Made in Monde. Les nouvelles frontières de l'économie mondiale, Paris, Seuil, 357 p.

Blum O. (2003), «Le déclassement à l'embauche : les diverses approches ", ministère de la Jeunesse, de l'Éducation nationale et de la Recherche, $C P C D o c u$ ments, $\mathrm{n}^{\circ} 6,51 \mathrm{p}$.

Bouffartigue P. (1994), « La socialisation professionnelle de jeunes BTS-DUT. Entre diplôme et statut, des identités incertaines ", Formation Emploi, $\mathrm{n}^{\circ} 45$, janvier-mars, pp. 3-23.

Coriat B. (1990), L'atelier et le robot, Paris, Christian Bourgois Éditions, 303 p.

Couppié Th., Giret J.-F., Lopez A. (2005), «Des formations initiales aux premiers emplois: une correspondance plutôt mal assurée », in J.-F. Giret, A. Lopez, J. Rose, Des formations pour quels emplois? Paris, La Découverte, "Recherches», pp. 79-96.

Coutrot Th. (1999), Critique de l'organisation $d u$ travail, Paris, La Découverte, «Repères », 120 p.

Dauty F., Lemistre Ph., Vincens J. (2006), « Le sens, la portée et le devenir de la nomenclature des "niveaux de formation" ", ministère de l'Éducation nationale, de l'Enseignement supérieur et de la Recherche, DGES, CPC Documents, $\mathrm{n}^{\circ} 2,114 \mathrm{p}$.

Demongeot A., Leprévost E (2008), « L'apprentissage : une voie de formation attractive, entre tradition et mutation », ministère de l'Éducation nationale, Note d'information de la Direction de l'Évaluation, de la Prospective et de la Performance, $\mathrm{n}^{\circ}$ 08.33, décembre, $6 \mathrm{p}$.

Enquête \& Opinion/UGICT-CGT (2006), Étude quantitative "Baromètre des professions intermédiaires", résultats détaillés, Metz, juillet, $100 \mathrm{p}$.

Fournié D., Guitton Ch. (2008), « Des emplois plus qualifiés, des générations plus diplômées : vers une modification des normes de qualification ", BrefCéreq, ${ }^{\circ} 252$, mai, $4 \mathrm{p}$.

Gambier D., Vernières M. (1991, 3e éd.), Le marché du travail, Paris, Économica, 231 p.

Giret J.-F., Hatot Ch. (2001), « Mesurer le déclassement à l'embauche: l'exemple des DUT et des BTS », Formation Emploi, n ${ }^{\circ} 75$, pp. 59-73.

Giret J.-F., Lemistre Ph. (2005), « Declassement of the young people: towards a change of the value of diplomas?», Brussels Economic Review, Special Issue: Economics of Education and Human Resources, vol. 43.

Giret J.-F., Lopez A., Rose J. (2005), Des formations pour quels emplois? Paris, La Découverte, « Recherches », $279 \mathrm{p}$.

Kern H., Schumann M. (1989) (trad. Franç.), La fin de la division du travail ? La rationalisation dans la production industrielle, Paris, Éditions de la Maison des Sciences de l'Homme, $417 \mathrm{p}$.

Labruyère C. (2006), « La VAE, quels candidats pour quels diplômes ? », Bref-Céreq, n² 230, mai, 4 p.

Le Roux A. (2005), « La validation des acquis dans l'enseignement supérieur en $2004 »$, ministère de l'Éducation nationale, Note d'information de la Direction de l'Évaluation, de la Prospective et de la Performance, $\mathrm{n}^{\circ}$ 05-28, octobre, $6 \mathrm{p}$.

Marion I., Möbus M., Sigot J.-C. (2008), « Formation professionnelle continue et changement de poste dans l'entreprise ", Céreq, Net.Doc, $\mathrm{n}^{\circ} 38$, avril, $34 \mathrm{p}$.

Maurice M., Eyraud F., d'Iribarne A., Rychener F. (1985), Les entreprises en mutation dans la crise: apprentissage des technologies flexibles et émergence des nouveaux acteurs, Aix-en-Provence, Lest, $450 \mathrm{p}$. 
Moncel N. (2008), « Recrutement en entreprise : les débutants sont-ils victimes d'un tri trop sélectif? », Bref-Céreq, $\mathrm{n}^{\circ} 250$, mars, $4 \mathrm{p}$.

Möbus M. (2009), «Les professions intermédiaires dans les entreprises. Cadrage statistique », NEFCéreq, $\mathrm{n}^{\circ} 39$, janvier, $50 \mathrm{p}$.

Moreau G. (2008), «Apprentissage : une singulière métamorphose », Formation Emploi, ${ }^{\circ} 101$, janviermars, pp. 119-133.

Oiry E., d'Iribarne A. (2001), «La notion de compétences: continuités et changements par rapport à la qualification », Sociologie $d u$ Travail, $\mathrm{n}^{\circ} 1$, pp. 49-66.

Paradeise C., Lichtenberger Y. (2001), « Compétence, compétences », Sociologie du Travail, n ${ }^{\circ}$, pp. 33-48.

Paul J.-J., Rose J. (2008), Les relations formationemploi en 55 questions, Paris, Dunod, 350 p.

Reynaud J.-D. (2001), «Le management par les compétences : un essai d'analyse ", Sociologie $d u$ Travail, $\mathrm{n}^{\circ} 1$, pp. 7-32.

Revue Formation Emploi (2008), Regards croisés sur les relations formations-emploi, numéro Anniversaire, 101, janvier-mars, $255 \mathrm{p}$.

Saglio J. (1988) «Négociations de classifications et régulation salariale dans le système français de relations professionnelles ", Travail et Emploi, $\mathrm{n}^{\circ} 38,12$, pp. 53-63.

Tallard M. (2001), «L'introduction de la notion de compétence dans les grilles de classification : genèse et évolution ", Sociétés contemporaines, $\mathrm{n}^{\circ}$ 41-42, pp. 159-187.

Trouvé Ph. (1996-a), «La fin des contremaîtres traditionnels?», Revue Française de Sociologie, XXXVII, pp. 287-308

Trouvé Ph. (1996-b), « L'éternelle “professionnalisation" des formations initiales en France », in S. Roussillon, F. Bournois, J.-Y. Le Louarn (Éd.), Les enjeux de l'emploi : société, entreprises et individus, CNRS, coll. «Programme Rhône-Alpes Recherches en Sciences Humaines », pp. 227-246.

Trouvé Ph. (1997) Les agents de maîtrise à l'épreuve de la modernisation industrielle. Essai de sociologie d'un groupe professionnel, Paris, L'Harmattan, «Logiques sociales », $311 \mathrm{p}$.

Trouvé Ph. (2009), « La contribution des entreprises à la formation de leur encadrement intermédiaire. Un investissement sans retour? », in Education permanente, dossier: Peut-on former à la fonction d'encadrement?, $\mathrm{n}^{\circ} 178 / 1$, pp. 37-53.

Ughetto P. (2007), Faire face aux exigences $d u$ travail contemporain. Conditions de travail et management, Lyon, Éditions de l'Anact, 157 p.

Ughetto P. (2008), «L'employeur exigeant et ses conditions de légitimité », in F. Hubault (coord.), Évaluation $d u$ travail, travail d'évaluation, Toulouse, Octarès, coll. «Le travail en débat», pp. 83-94.

Veneau P., Personnaz E. (2002), «Les certificats de qualification paritaires de la métallurgie : une forme d'ajustement formation-emploi?», Travail et Emploi, $\mathrm{n}^{\circ}$ 90, avril, pp. 87-98.

Verdier E. (1995), « Politiques de formation des jeunes et marché du travail. La France des années quatre-vingts », Formation Emploi, ${ }^{\circ}$ 50, avril-juin, pp. 19-40.

Verdier E. (1996), «L'insertion professionnelle des jeunes "à la française": vers un ajustement structurel ? », Travail et Emploi, ${ }^{\circ}$ 4, La Documentation française, pp. 37-54.

Verdier E. (2001), «La France a-t-elle changé de régime d'éducation et de formation? », Formation Emploi, $\mathrm{n}^{\circ} 76$, numéro Spécial 30 ans du Céreq, octobre-décembre, pp. 11-34.

Zarifian Ph. (1999), Objectif compétence : pour une nouvelle logique, Paris, Éditions Liaisons, 229 p.

Zarifian Ph. (2001), Le modèle de la compétence, Paris, Éditions Liaisons, coll. «Entreprise \& Carrières », $114 \mathrm{p}$. 